\title{
Study of indigenous freshwater fish diversity of Bankura (West Bengal), India with special reference to Clarias batrachus
}

\section{Arindam Ganguly \\ Department of Microbiology, Vidyasagar University, Midnapore-721102 (West Bengal) India}

Amrita Banerjee

Department of Microbiology, Vidyasagar University, Midnapore-721102 (West Bengal), India

Asish Mandal

P.G. Department of Botany, Ramananda College, Bishnupur, Bankura-722122 (West Bengal), India

\section{Tapas Kumar Dutta}

Department of Zoology, Bankura Sammilani College, Bankura- 722102 (West Bengal), India

\section{Pradeep Kumar Das Mohapatra*}

Department of Microbiology, Raiganj University, Raiganj, Uttar Dinajpur- 733134 (West Bengal), India

*Corresponding author. E-mail: pkdmvu@gmail.com

\section{Abstract}

A study was conducted to determine the ichthyofaunal diversity in Bankura district of West Bengal. The area is traversed by major and minor rivers, feeder channels, numerous ponds, bills, reservoirs which have made this drought-trodden district as the highest producer of aquatic products within the state. Field survey was conducted in randomly selected local markets covering twenty-two blocks of Bankura. Fish sampling was carried out using various conventional fishing gears. The entire region harbours a wide variety of aquatic fishes. A total number of 92 indigenous fish species belonging to 30 families were identified during this study. The Cyprinidae family dominated the population with its 36 varieties followed by Channidae, Siluridae and Bagridae. Sonamukhi block situated in Shali basin; Sarenga and Raipur of Kangsabati basin; Dwarkeshwar and seven bundhsenriched Bishnupur were the major habitats of small indigenous fishes. This paper also denotes presence of 12 globally endemic freshwater fish species viz. Hypophthalmichthys molitrix, Cyprinus carpio, Labeo nandina, Tor khudree, Chitala chitala, Bagarius bagarius, Wallago attu, Ompok pabda, Ailia coila, Anguilla bengalensis, Parambassis lala, Oreochromis mossambicus. The substitution of native Clarias batrachus with invasive African catfish $C$. gariepinus was observed in various local markets. Some traders even promote illicit farming of this banned species for their own profit. As a consequence, the indigenous, nutritionally-enriched $C$. batrachus is becoming more endangered.

Keywords: Clarias batrachus, Fish diversity, Small indigenous fish

\section{Article Info}

DOI:10.31018/jans.v10i4.1892 Received: September 13, 2018 Revised: October 20, 2018 Accepted: October 29, 2018

\section{How to Cite}

Ganguly, A. et al. (2018). Study of indigenous freshwater fish diversity of Bankura (West Bengal), India with special reference to Clarias batrachus. Journal of Applied and Natural Science, 10(4): $1162-1172$

\section{INTRODUCTION}

The district of Bankura $\left(22^{\circ} 38^{\prime}\right.$ to $23^{\circ} 38^{\prime} \mathrm{N}$ and $86^{\circ} 36^{\prime}$ to $\left.87^{\circ} 46^{\prime} \mathrm{E}\right)$, over a massive geographical area of $6,882 \mathrm{~km}^{2}$, enjoys lush green views combined with uneven plateau which links plains of Bengal to the peninsular mass of Chota Nagpur. The area is beset with intermittent dense forest, red ferruginous laterite soil and DamodharDwarkeshwar-Shilabati-Kangsabati riverine network. The rivers of the district get a lease of life with monsoon rains. The same can be attributed to the reservoirs and ponds of the region. The aquaculture farms (14400 ha) are thus mainly dependent upon the supply of groundwater along with rain water throughout the year. The climate is tropical dry sub-humid with an annual rainfall of 1100 to $1400 \mathrm{~mm}$. This combination of seasonal streams, rain-soaked natural ponds, aquaculture farms, rivers and their feeder channels are the rich sources $(52341 \mathrm{Ha})$ of freshwater indigenous fishes (Anonymous, 2016).

Small indigenous fishes (which attain a maximum length of $25-30 \mathrm{~cm}$ in mature stage) comprise a large portion of fish biomass and a major contributor of nutritional security to the rural people. Fish 
circulates essential nutrients between the littoral, limnetic, profundal and benthic zones. Mohanty et al. (2013) reported that these indigenous fishes are the source of vital proteins (Xenentodon cancila, Ailia coila) and micro-nutrients such as Calcium (Puntius sophore, Gudusia chapra, Chanda nama), Zinc (Mystus tengra, Chela bacaila), iron (Osteobrama cotio, Mystus vittatus), as well as vitamins (Esomus danricus, Parambassis ranga) and fatty acids (Amblypharyngodon mola, Channa punctata). Thus, they play a major role in community nutrition in a district like Bankura where about $41.52 \%$ families are thriving under poverty (Anonymous, 2016).

Clarias batrachus (Linn.) is a widely recognized small indigenous fish by virtue of its nutritional richness. It is presently thriving in Southern Asia at a merely vulnerable state despite its high demand among consumers. Siltation of natural breeding grounds, intermittent periods of drought, indiscriminate use of pesticides in paddy fields, industrial effluents and illicit fishing of juveniles has made the situation more hostile (Dhara and Saha, 2013). Some traders often substitute $C$. batrachus with invasive alien catfish $C$. gariepinus in the local name of 'Dēśi Māgur' due to their morphological resemblance (Khedkar et al., 2015). The biological invasion of $C$. gariepinus in natural water bodies can be a potential threat to biodiversity due to its frenzied feeding behavior (Radhakrishnan et al., 2011). It has become a burning issue in South China, Brazil and India to protect endemic and endangered small indigenous fishes from prolific predatory nature of $C$. gariepinus (Khedkar et al., 2015).

To create conservation strategy as a whole, knowledge of the occurrence of the species, present status and distribution range is essential. Mapping of fish diversity area is also important in view of conservation of those fish species in natural habitats. The present investigation aims to study the ichthyofaunal diversity of Bankura district along with a search of threatened, endemic and vulnerable species. Special emphasis on $C$. batrachus was also given to verify biological invasion of African catfish C. gariepinus in local water bodies.

\section{MATERIALS AND METHODS}

Field survey: Field sampling and survey were conducted during 2017-2018 in randomly chosen waterbodies and local fish markets of twenty-two community development blocks of bankura district (Fig. 1). Primary data was collected from the fishermen and fish-sellers whereas secondary data was obtained from the concerned authorities, Govt. agencies and research publications. The graphical representation and statistical analysis of data was carried out using Microsoft Office Excel software. Garmin eTrex Vista Hcx Global Position- ing system device has been utilized to obtain specific location status. The Geographic information system (GIS) based spatial distribution of fish diversity was analyzed through Inverse-distance weighting (IDW) spatial interpolation method by using ArcGIS 10.2 (http://www.esri.com) software. Collection of samples: Various conventional fishing gears (Fig. 2) were employed to collect specimen with the help of fish farmers from the study area.

It included Ghuni (Used to catch bottom dwelling fishes like Trichogaster chuna, Anabas testudineus etc.), Palu'i (Used to catch fishes in slime), Dughārē (Used to catch small indigenous fishes in shallow river), Baraśi (Used to catch small and medium sized fishes), Cāp Jāl (Used to catch small fishes), Drag net (Used to catch carps, small indigenous fishes of a pond), Ghun Jāl, Phāmindi, Wheel chip, Jhim chip, Ṭōgi, Jațā (Used to catch C. orientalis, Monopterus cuchia, Clarias batrachus etc.), Larkā (Used to catch C. striata), Kēmncā (Used to catch fishes like C. striata) etc. The samples were immediately preserved in 4-6\% formalin and brought to laboratory for further identification (Roy et al., 2013). Specimens were precisely recorded by photography. Identification and subsequent taxonomic classification of those samples were done according to Talwar and Jhingran (1991) and FishBase (2016). The International Union for Conservation of Nature (IUCN, 2018) red list of threatened species was followed to evaluate the present conservation status of the species.

\section{RESULTS}

A total number of 92 indigenous freshwater fish species belonging to 30 families under 10 orders were identified in the study area. The GIS-based fish diversity map showed 11 different regions (by

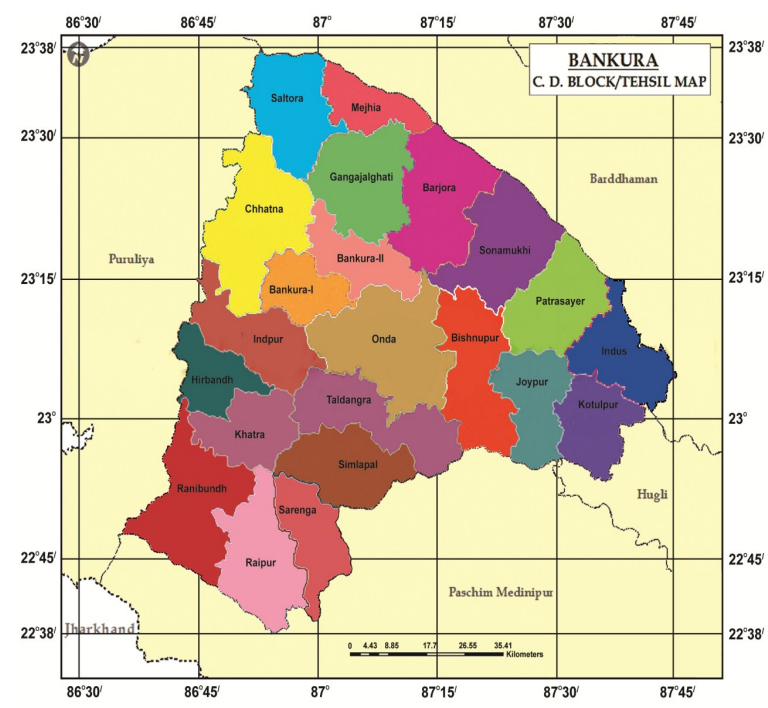

Fig. 1. Geographical map of the study area. 
Ganguly, A. et al. / J. Appl. \& Nat. Sci. 10 (4): 1162 -1172 (2018)

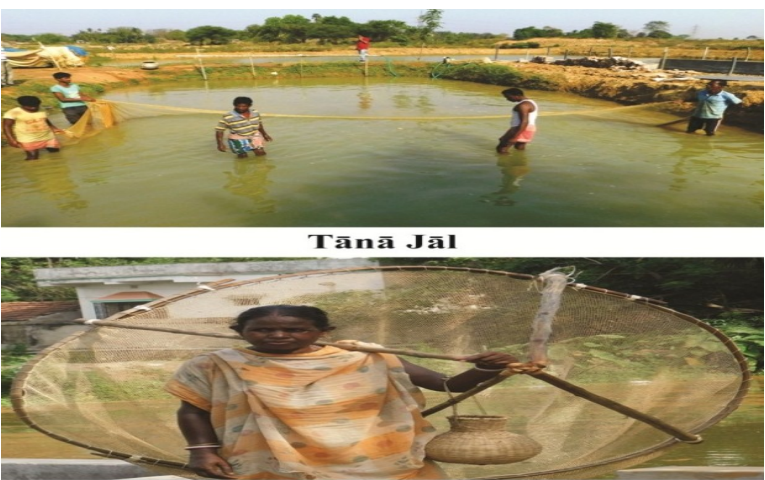

C $\overline{\mathbf{a}} \mathbf{p} \mathrm{J} \overline{\mathbf{a}} \mathbf{I}$

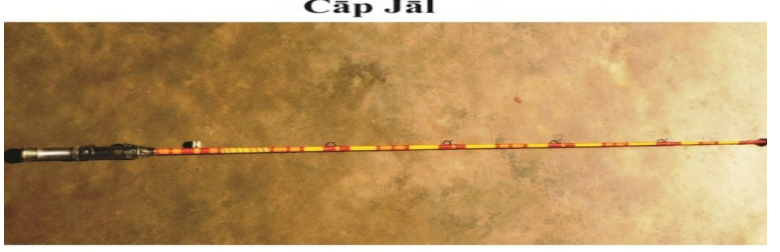

Jhima chipa

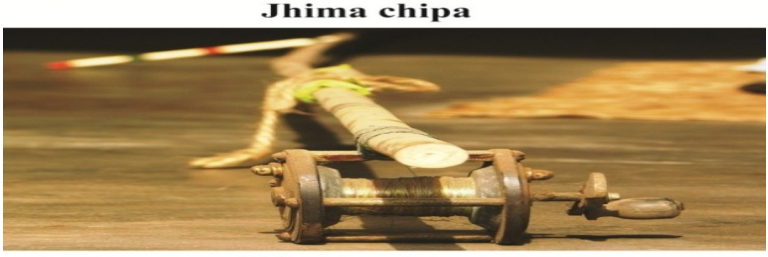

Wheel chipa

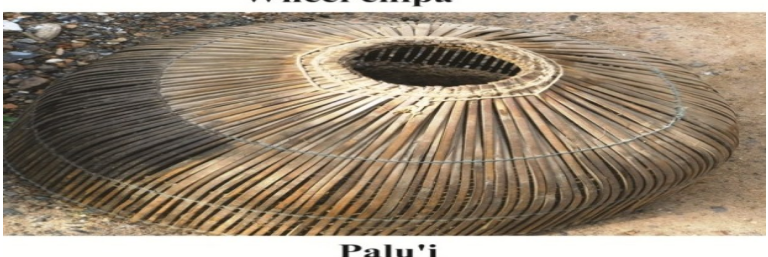

Palu'i
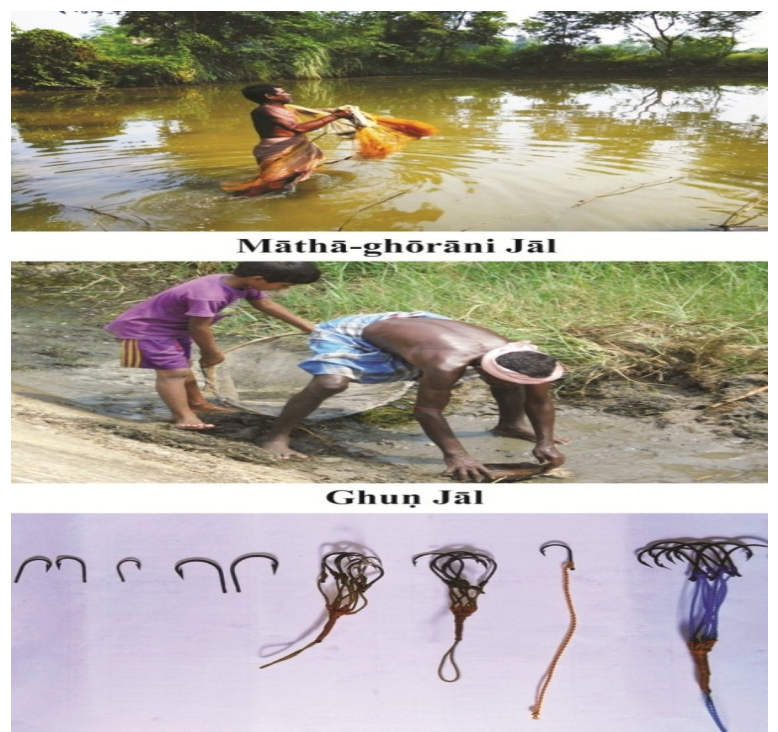

Barasi

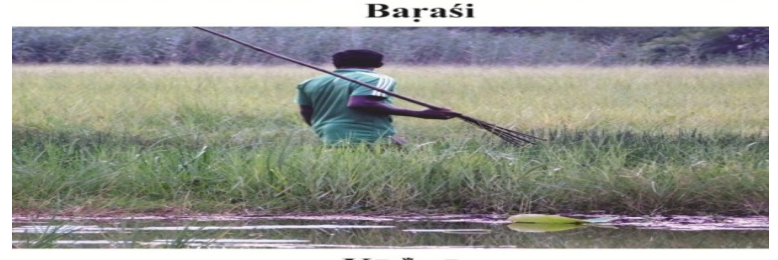

Kēñ cā

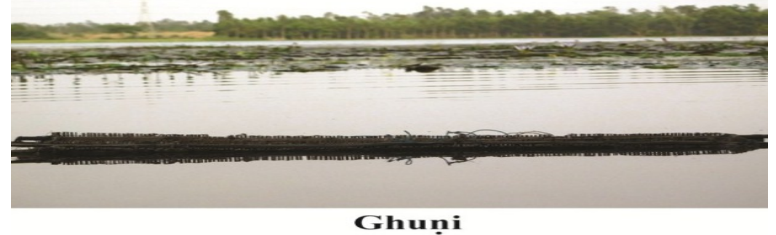

Fig. 2. Fishing gears used to collect fish during the study.

using equal interval method) of spices richness. Bishnupur, Bankura-II, Raipur, Barjora, Hirbandh and Saltora blocks were very rich in the context of species availability of fish (Fig. 3).

The entire northern and southern part of Bankura District is also significantly rich where more than 50 species are available in general. Due to rugged topography and impermeable soil layer, a greater portion of Indus, Chhatna, and Taldangra blocks have only a few water bodies and subsequently lower fish diversity. The order-wise fish diversity has illustrated predominance of Cypriniformes $(42.39 \%)$, Siluriformes $(20.65 \%)$ and Perciformes $(21.74 \%)$ over the area (Fig. 4). Anguilliformes and Tetraodontiformes were least observed with only single species under each of them. Sarenga and Raipur blocks of Kangsabati basin were the major habitats of small indigenous fishes. Ramsagar of Onda block was identified as a potential breeding centre of major carps being the destination of several hatcheries for qualitative spawns.

The Cyprinidae family dominated the population with 36 varieties throughout the district followed by
Channidae (6), Siluridae (4) and Bagridae (4). Block wise distribution (Table 1) showed maximum diversity in the blocks Bankura-I (69), Raipur (67), Barjora (66), Saltora (65) and Sarenga (65) ranging from economically significant species such as Dēśī Māgur (Clarias batrachus), Śingī (Heteropneutes fossilis), Dēśī kō'i (Anabas testudineus) to rare or near threatened Maulā (Anguilla bengalensis), Kānāghōghōr (Bagarius bagarius) or Bōȳāla (Wallago attu).

A large number of species has newly been revealed in this area through this study. The distribution pattern indicated presence of several threatened, endemic and vulnerable species in rural waterbodies (Table 2). The result was slightly different from earlier reports of maximum diversity at Onda, Hirbundh and Raipur region. Roy et al. (2013) reported rapid declining of $C$. chitala and O. pabda in Bankura district whereas the present study indicated considerable recovery and more availability of those species in that region. Additionally, the presence of endangered species Tor khudree in Bankura district was not previously reported. 
Ganguly, A. et al. / J. Appl. \& Nat. Sci. 10 (4): 1162 -1172 (2018)

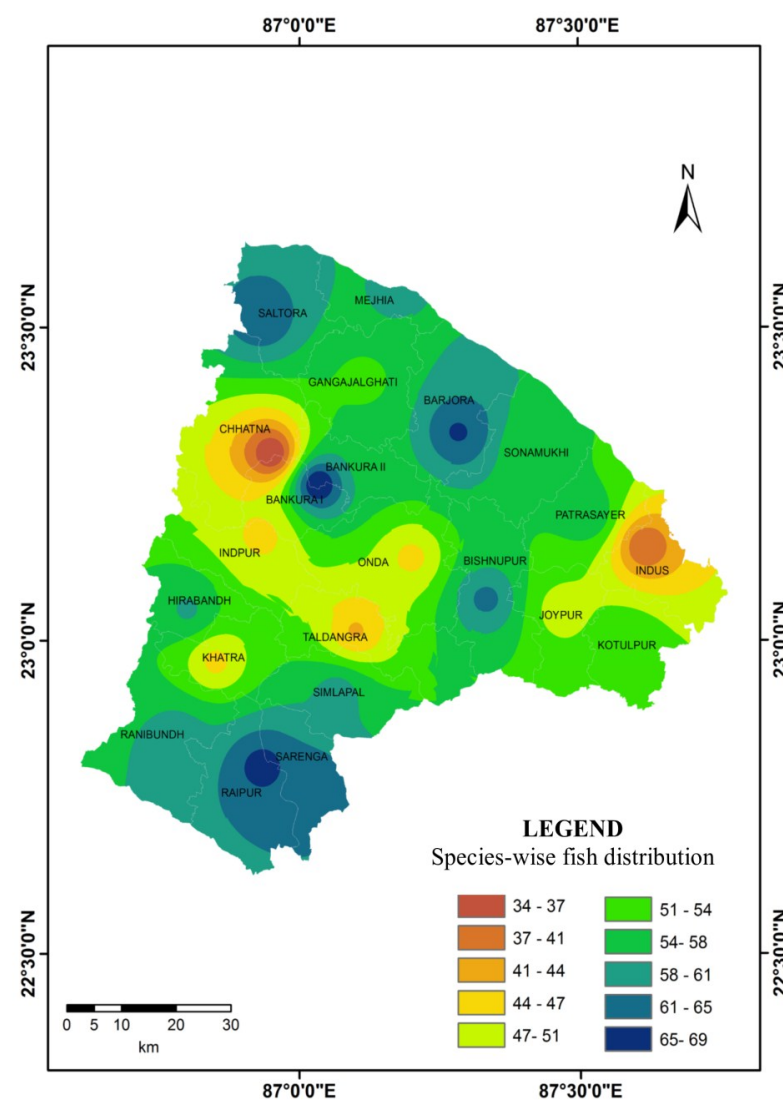

Fig. 3. Spatial distribution of fish diversity of Bankura district.

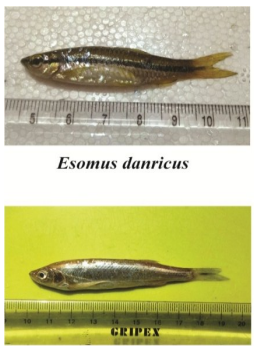

Salmophasia bacaila
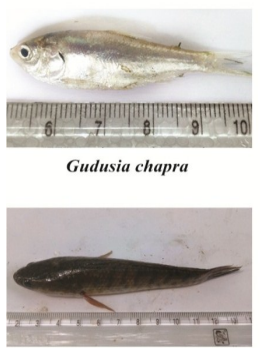

Channa punctata

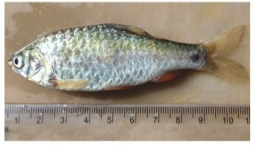

Puntius ticto

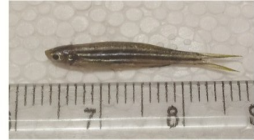

Danio rerio

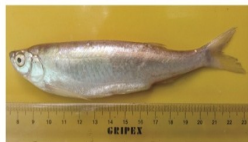

Salmostoma bacaila

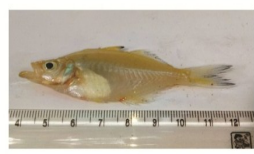

Chanda nama

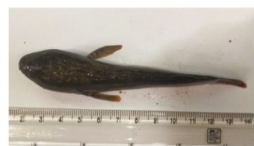

Channa orientalis

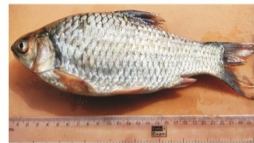

Puntius sarana

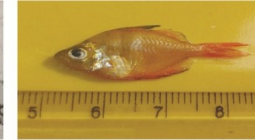

Parambassis lala

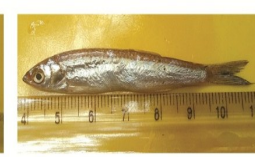

Securicula gora
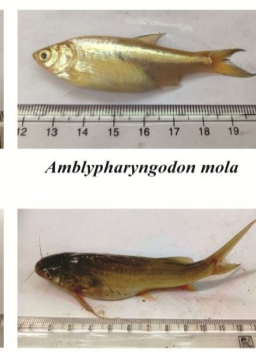

Mystus tengra

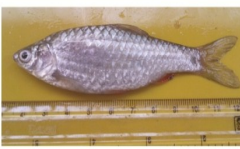

Puntius sophore

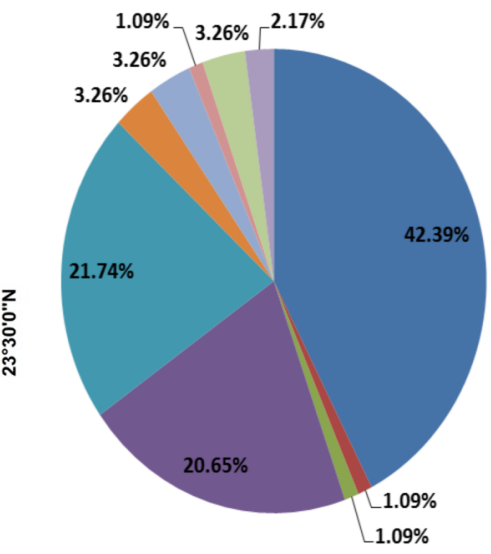

- Cypriniformes

- Anguilliformes

- Tetraodontiformes

- Siluriformes

- Perciformes

- Clupeiformes

Mugiliformes

m Beloniformes

॥ynbranchiformes

m Osteoglossiformes

Fig. 4. Order wise fish distribution of Bankura, West Bengal.

A large diversity of small indigenous fishes (Table 3) was observed in local markets (Bankura-l, Raipur, Barjora, Saltora, Hirbandh and Sarenga blocks) of Bankura district near rivers basin. Amblypharyngodon mola, Esomus danricus, Sal-

z mostoma bacaila, Heteropneustes fossilis, Clarias batrachus, Mystus tengra, Chanda nama, Channa orientalis and Channa punctata were mostly consumed. These species (Fig. 5) are either obtained by fishing from nearby water bodies or caught from natural ponds predominantly in monsoon.

C. batrachus has a high demand throughout the Bankura district. It is majorly been found once or

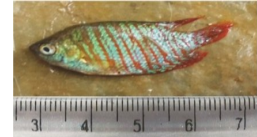

Trichogaster lalius

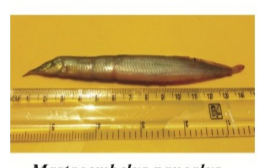

Mastacembelus pancalus

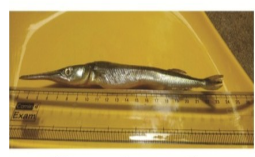

Xenentodon cancila

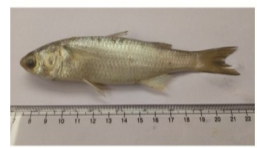

Liza parsia

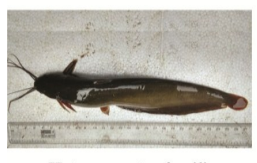

Heteropneutes fossilis

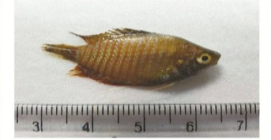

Trichogaster fasciata

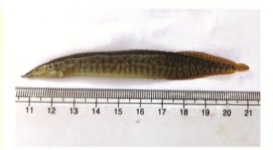

Mastacembelus armatus

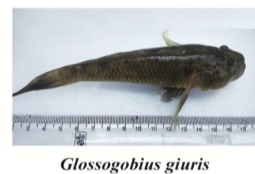

Glossogobius giuris

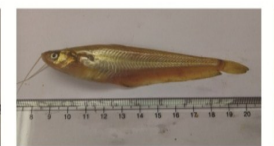

Ompok pabda

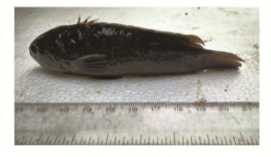

Anabas testudineus

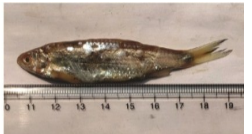

Aspidoparia jaya

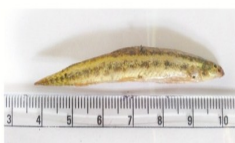

Lepidocephalus guntea
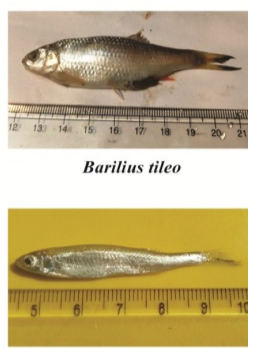

Ailia coila

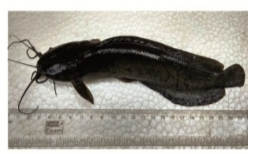

Clarias batrachus

Fig. 5. Some freshwater small indigenous fishes of Bankura district. 
Ganguly, A. et al. / J. Appl. \& Nat. Sci. 10 (4): 1162 -1172 (2018)

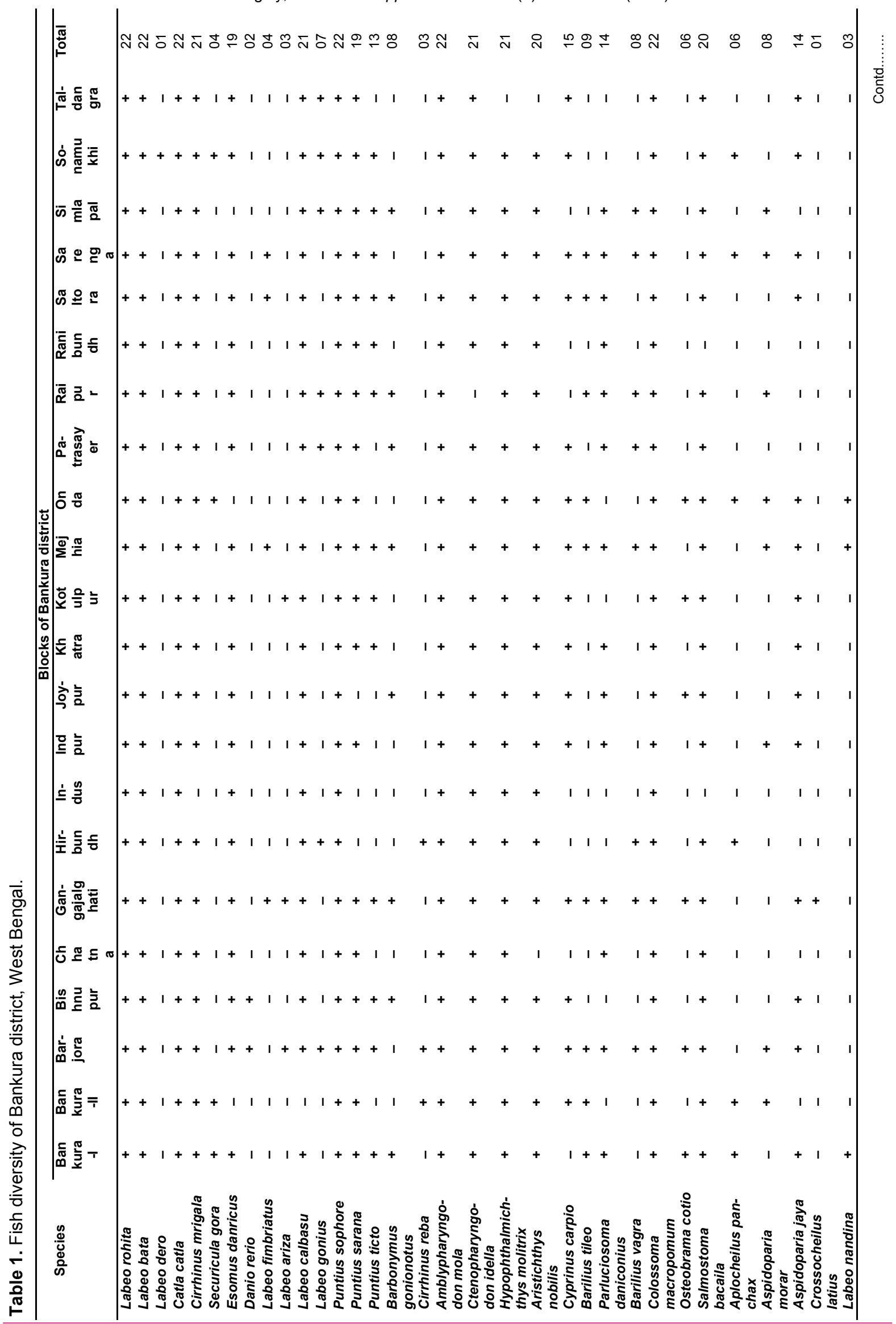


Ganguly, A. et al. / J. Appl. \& Nat. Sci. 10 (4): 1162 -1172 (2018)

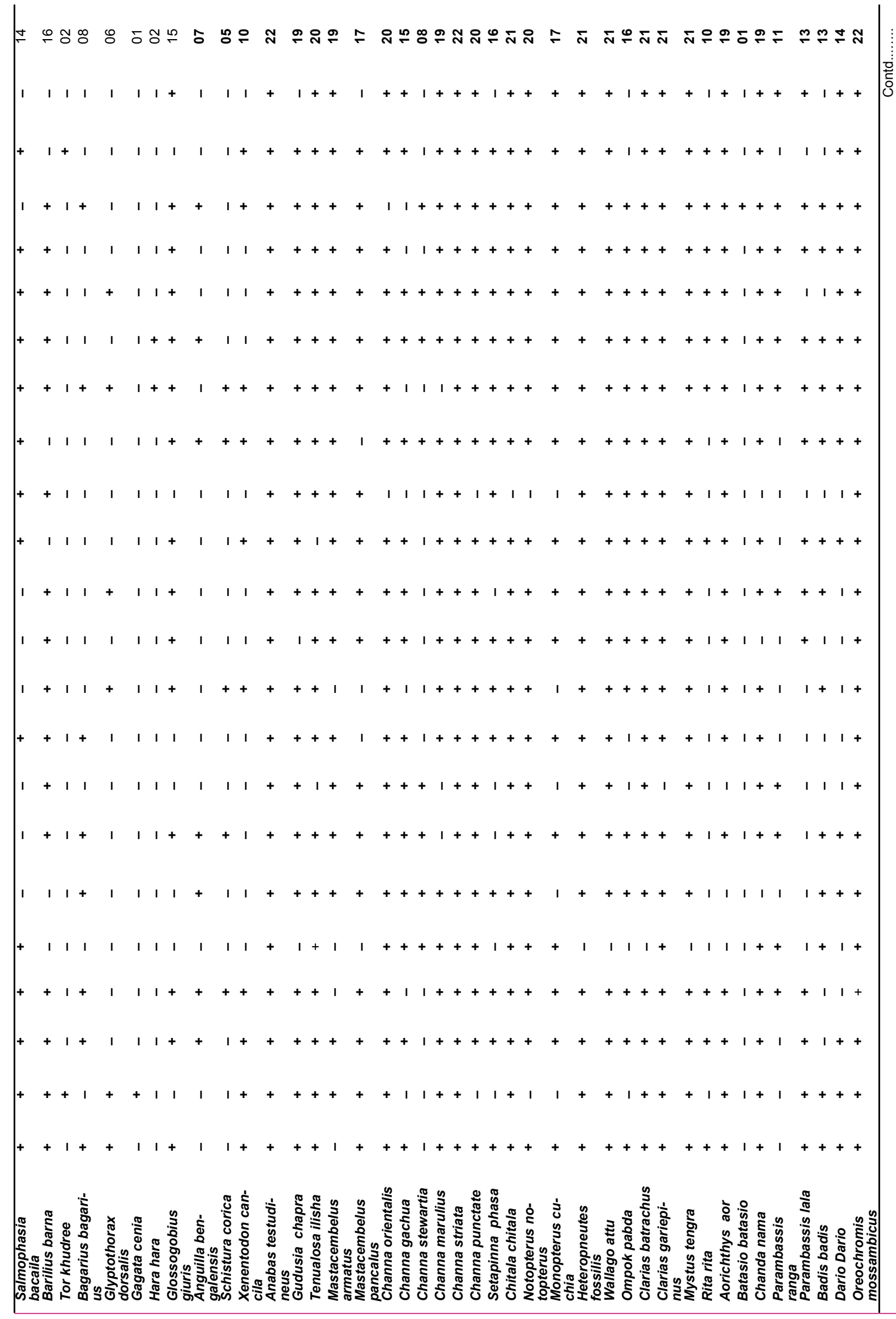




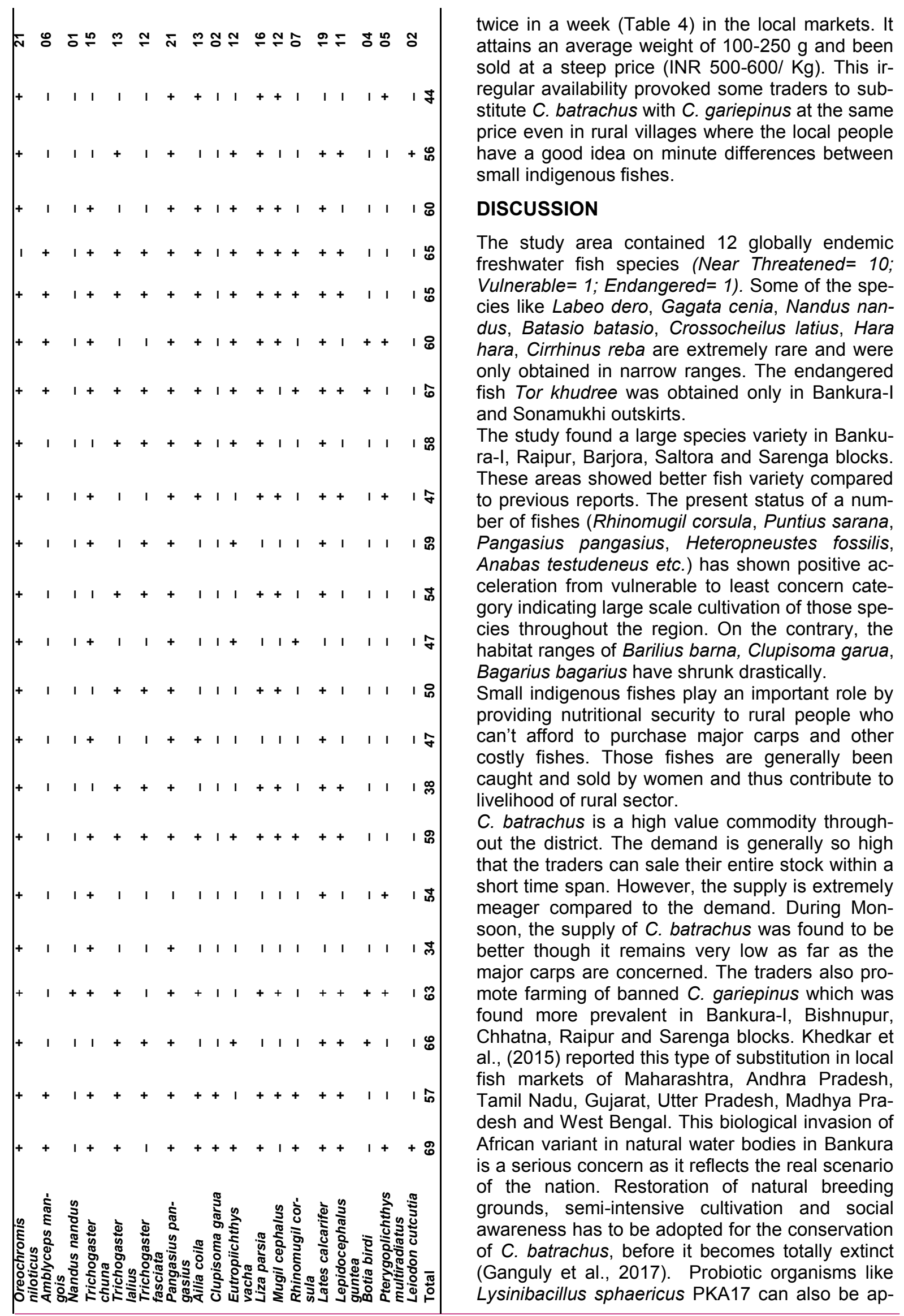


Ganguly, A. et al. / J. Appl. \& Nat. Sci. 10 (4): 1162 -1172 (2018)

Table 2.Taxonomic position and conservation status of different fishes of Bankura district, West Bengal.

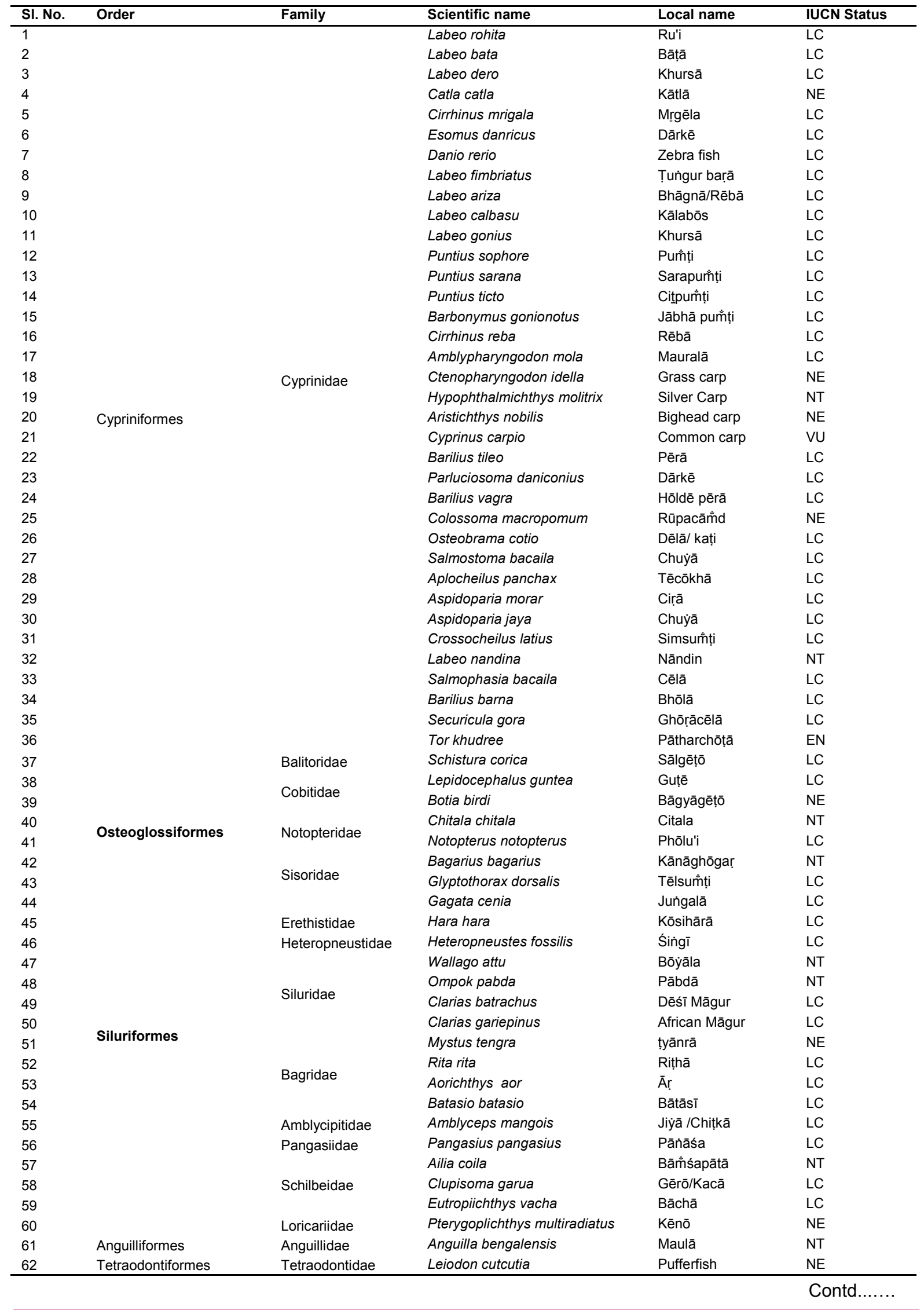


Ganguly, A. et al. / J. Appl. \& Nat. Sci. 10 (4): 1162 -1172 (2018)

\begin{tabular}{|c|c|c|c|c|c|}
\hline 63 & \multirow{21}{*}{ Perciformes } & Gobiidae & Glossogobius giuris & Bhalkōrā/ Bēlē & $\mathrm{LC}$ \\
\hline 64 & & Anabantidae & Anabas testudineus & Dēśī kō'i & $\mathrm{DD}$ \\
\hline 65 & & \multirow{6}{*}{ Channidae } & Channa orientalis & Cyām & $\mathrm{NE}$ \\
\hline 66 & & & Channa gachua & Śiśircyāṃ & LC \\
\hline 67 & & & Channa striata & Śōl & LC \\
\hline 68 & & & Channa stewartii & Tēlcyāmi & LC \\
\hline 69 & & & Channa punctata & Lyāțā & LC \\
\hline 70 & & & Channa marulius & Śāl & $\mathrm{LC}$ \\
\hline 71 & & \multirow{3}{*}{ Ambassidae } & Chanda nama & Cāmỏdakōmrṇā & LC \\
\hline 72 & & & Parambassis ranga & Cāmảdakōṃ̆rāā & LC \\
\hline 73 & & & Parambassis lala & Lāl Cām̆dakōm̆rnā & NT \\
\hline 74 & & \multirow[b]{2}{*}{ Badidae } & Badis badis & Kalōpum̆ți & LC \\
\hline 75 & & & Dario dario & Lālapum̋tți & DD \\
\hline 76 & & \multirow{2}{*}{ Cichlidae } & Oreochromis mossambicus & Tēlāpiȳā & NT \\
\hline 77 & & & Oreochromis niloticus & Nilanțikā & $\mathrm{NE}$ \\
\hline 78 & & \multirow[t]{2}{*}{ Nandidae } & Nandus nandus & N'yādōs & LC \\
\hline 79 & & & Trichogaster chuna & Cūnā & LC \\
\hline 80 & & \multirow[t]{2}{*}{ Osphronemidae } & Trichogaster lalius & Khōlsē & LC \\
\hline 81 & & & Trichogaster fasciata & Khōlsē & LC \\
\hline 82 & & Latidae & Lates calcarifer & Bhēṭki & $\mathrm{NE}$ \\
\hline 83 & & \multirow{2}{*}{ Clupeidae } & Tenualosa ilisha & Iliś & LC \\
\hline 84 & \multirow[t]{2}{*}{ Clupeiformes } & & Gudusia chapra & Khaỳarā & LC \\
\hline 85 & & Engraulidae & Setipinna phasa & Phāsā & LC \\
\hline 86 & \multirow{3}{*}{ Mugiliformes } & \multirow{3}{*}{ Mugilidae } & Liza parsia & Pārśē & $\mathrm{NE}$ \\
\hline 87 & & & Mugil cephalus & Pārśē & LC \\
\hline 88 & & & Rhinomugil corsula & Kyaklās & LC \\
\hline 89 & Beloniformes & Belonidae & Xenentodon cancila & Gāntārāā & LC \\
\hline 90 & \multirow{3}{*}{ Synbranchiformes } & Synbranchidae & Monopterus cuchia & Kucē & LC \\
\hline 91 & & \multirow{2}{*}{ Mastacembelidae } & Mastacembelus armatus & Pyam̆kāl & $\mathrm{LC}$ \\
\hline 92 & & & Mastacembelus pancalus & Pyam̌kāl & $\mathrm{NE}$ \\
\hline
\end{tabular}

$\mathrm{NE}=$ Not Evaluated; $\mathrm{DD}=$ Data Deficient; $\mathrm{LC}=$ Least Concern; NT= Near Threatened; VU= Vulnerable; EN=Endangered

Table 3. Small indigenous fishes of Bankura district, West Bengal.

\begin{tabular}{|c|c|c|}
\hline SI. No. & Scientific name & Local name \\
\hline 1 & Esomus danricus (Hamilton, 1822) & Dārkē \\
\hline 2 & Danio rerio (Hamilton, 1822) & Zebra fish \\
\hline 3 & Puntius sophore (Hamilton, 1822) & Pum̆tți \\
\hline 4 & Puntius ticto (Hamilton, 1822) & Citpuñuți \\
\hline 5 & Amblypharyngodon mola (Hamilton, 1822) & Mauralā \\
\hline 6 & Barilius tileo (Hamilton, 1822) & Pērā \\
\hline 7 & Parluciosoma daniconius (Hamilton, 1822) & Dārkē \\
\hline 8 & Barilius vagra (Hamilton, 1822) & Hōldē pērā \\
\hline 9 & Osteobrama cotio (Hamilton, 1822) & Dēlā/ kați \\
\hline 10 & Salmostoma bacaila (Hamilton, 1822) & Chuỹā \\
\hline 11 & Aplocheilus panchax (Hamilton, 1822) & Tēcōkhā \\
\hline 12 & Aspidoparia morar (Hamilton, 1822) & Cirā \\
\hline 13 & Aspidoparia jaya (Hamilton, 1822) & Chuȳā \\
\hline 14 & Crossocheilus latius (Hamilton, 1822) & Simsum̆ți \\
\hline 15 & Salmophasia bacaila (Hamilton, 1822) & Cēlā \\
\hline 16 & Barilius barna (Hamilton, 1822) & Bhōlā \\
\hline 17 & Securicula gora (Hamilton, 1822) & Ghōrāāēlā \\
\hline 18 & Glyptothorax dorsalis (Vinciguerra, 1890) & Tēlsum̆ți \\
\hline 19 & Hara hara (Hamilton, 1822) & Kōsihārā \\
\hline 20 & Gagata cenia (Hamilton, 1822) & Jungalā \\
\hline 21 & Glossogobius giuris (Hamilton, 1822) & Bhalkōrā/Bēlē \\
\hline 22 & Schistura corica (Hamilton, 1822) & Sālgēțō \\
\hline 23 & Botia birdi (Chaudhuri, 1909) & Bāgyāgēțō/Birdi Loach \\
\hline 24 & Anabas testudineus (Bloch, 1792) & Dēśī kō'i \\
\hline 25 & Gudusia chapra (Hamilton, 1822) & Khaỳarā \\
\hline 26 & Mastacembelus pancalus (Hamilton, 1822) & Pyam̌́kāl \\
\hline 27 & Channa orientalis (Bloch and Schneider, 1801) & Cyām \\
\hline 28 & Channa gachua (Hamilton, 1822) & Śiśircyām \\
\hline 30 & Channa stewartii (Playfair, 1867) & Tēlcyām \\
\hline 31 & Channa punctata (Bloch, 1793) & Lyāțā \\
\hline 32 & Setapinna phasa (Hamilton, 1822) & Phāsā \\
\hline 33 & Eutropiichthys vacha (Hamilton, 1822) & Bāchā \\
\hline
\end{tabular}


Ganguly, A. et al. / J. Appl. \& Nat. Sci. 10 (4): 1162 -1172 (2018)

\begin{tabular}{|c|c|c|}
\hline 34 & Heteropneustes fossilis (Bloch, 1794) & Śingī \\
\hline 35 & Ompok pabda (Hamilton, 1822) & Pābdā \\
\hline 36 & Clarias batrachus (Linnaeus, 1758) & Dēśī Māgur \\
\hline 37 & Mystus tengra (Hamilton, 1822) & țyānrā \\
\hline 38 & Batasio batasio (Hamilton, 1822) & Bātāsī \\
\hline 39 & Chanda nama (Hamilton, 1822) & Cām̆dakōm̆rāā \\
\hline 40 & Parambassis ranga (Hamilton, 1822) & Cām̆dakōm̆rịā \\
\hline 41 & Parambassis lala (Hamilton, 1822) & Lāl Cām̆dakōmñrāa \\
\hline 42 & Badis badis (Hamilton, 1822) & Kalōpum̆tți \\
\hline 43 & Dario dario (Hamilton, 1822) & Lālapum̆ți \\
\hline 44 & Amblyceps mangois (Hamilton, 1822) & Jiȳā /Chițkā \\
\hline 45 & Nandus nandus (Hamilton, 1822) & N'yādōs \\
\hline 46 & Trichogaster chuna (Hamilton, 1822) & Cūnā \\
\hline 47 & Trichogaster lalius (Hamilton, 1822) & Khōlsē \\
\hline 48 & Trichogaster fasciata (Bloch and Schneider, 1801) & Khōlsē \\
\hline 49 & Ailia coila (Hamilton, 1822) & Bām̆śapātā \\
\hline 50 & Liza parsia (Hamilton, 1822) & Pārśē \\
\hline 51 & Mugil cephalus (Hamilton, 1822) & Pārśē \\
\hline 52 & Lepidocephalus guntea (Hamilton, 1822) & Guțē \\
\hline 53 & Xenentodon cancila (Hamilton, 1822) & Gāntārāā \\
\hline
\end{tabular}

Table 4. Survey on Clarias batrachus of Bankura district, West Bengal.

\begin{tabular}{lllll}
\hline SI. No. & Blocks & Frequency & Price (INR/Kg) & Average Weight (g) \\
\hline 1 & Bankura-I & Seasonal & $550-600$ & $200-250$ \\
2 & Bankura-II & Seasonal & $600-650$ & $130-150$ \\
3 & Barjora & Once or twice in a week & 600 & $100-120$ \\
4 & Bishnupur & Once in a week & $550-600$ & $100-150$ \\
5 & Chhatna & Rare & 600 & $230-250$ \\
6 & Gangajalghati & Weekly & 500 & $230-250$ \\
7 & Hirbundh & Available & $400-500$ & $200-250$ \\
8 & Indus & Seasonal & $650-700$ & $200-250$ \\
9 & Indpur & Weekly & $400-450$ & $250-300$ \\
10 & Joypur & Seasonal & $500-550$ & $150-200$ \\
11 & Khatra & Infrequent & $500-550$ & $250-300$ \\
12 & Kotulpur & Infrequent & 550 & $150-200$ \\
13 & Mejhia & Once in a month & $600-800$ & $200-250$ \\
14 & Onda & Weekly & $400-450$ & $150-180$ \\
15 & Patrasayer & Regular & $400-450$ & $150-180$ \\
16 & Raipur & Seasonal & $500-600$ & $250-300$ \\
17 & Ranibundh & Regular & 400 & $150-250$ \\
18 & Saltora & Infrequent & 500 & $100-150$ \\
19 & Sarenga & Infrequent & 600 & $250-300$ \\
20 & Simlapal & Seasonal & 450 & $200-250$ \\
21 & Sonamukhi & Rare & $300-400$ & $100-150$ \\
22 & Taldangra & Seasonal & 500 & $200-250$ \\
\hline
\end{tabular}

INR= Indian Rupee

plied at proper dose level to enhance the growth and disease-resistance of $C$. batrachus as suggested earlier (Ganguly et al., 2018).

\section{Conclusion}

The study provided a new perspective of ichthyofaunal diversity of Bankura district in West Bengal in India. It revealed a wide variety of species which constitutes a significant portion $(53.80 \%)$ of freshwater indigenous fishes of the state. The area is predominated by the order Cypriniformes $(42.39 \%)$ which includes the family Cyprinidae, Balitoridae and Cobitidae. The Siluriformes and Perciformes also majorly contribute towards biodiversity. The riverine civilization, suitable climate, soil profile and edge effects have enriched the ichthyofaunal diversity of the district. The inland fish and fisheries are making substantial contribution towards rural economy and women empowerment. A major portion of rural-backward people rely upon inland fisheries as a source of nutritional and economic security. However, the restoration of indigenous fishes has often grossly been underestimated. Rapid industrialization, indiscriminate use of chemical fertilizers and pesticides in adjacent agricultural fields more worsen the situation. There are a good number of globally threatened fishes still thriving in this geopolitical area that demand our utmost attention right now. Intensive study equipped with advance technologies needs to be carried out. The threatened and endangered species must be exclusively cultivated in their nat- 
ural habitats free of any industrial and agricultural pollutants for the conservation of the species. The nutritional value of all freshwater indigenous fishes should also be assessed. The restoration of indigenous $C$. batrachus must be prioritized through semi-intensive cultivation. Social awareness among common people and fish-farmers has to be intricated. The Government and other stakeholders must also step up to restore the fish diversity and rejuvenate old waterways. Serious involvement of all concerned authorities is highly needed to develop an enriched fish profile of the entire district.

\section{ACKNOWLEDGEMENTS}

The first author is thankful to the students under project work in the Department of Microbiology of Bankura Sammilani College, West Bengal, India for their commitment in survey.

\section{REFERENCES}

1. Dhara, K., and Saha, N.C. (2013). Controlled Breeding of Asian Catfish Clarias batrachus using Pituitary Gland Extracts and Ovaprim at different Temperatures, Latency Periods and their Early Development. Journal of Aquaculture Research and Development, 4(4), 1-9. https://doi.org/10.4172/2155-9546.1000186

2. Ganguly, A., Mandal, A., Khan, M.A., Dutta, T.K., Raha, S. and Das Mohapatra, P.K. (2017). Study of Physico-chemical Parameters, Planktonic Diversity and Bacterial Load of Clarias batrachus Cultivation
Pond at Bankura, WB, India. International Research Journal of Biological Sciences, 6(12): 23-34.

3. Ganguly, A., Banerjee, A., Mandal, A. and Das Mohapatra, P.K. (2018). Optimization of effective dose of a newly isolated probiotic bacteria for growth and disease resistance of Clarias batrachus (linn.). Research Journal of Life Sciences, Bioinformatics, Pharmaceutical and Chemical Sciences, 4(4) : 344$354 \mathrm{https}: / /$ doi.org/10.26479/2018.0404.30

4. IUCN Red List of Threatened Species http:// www.iucnredlist.org (Access on June, 2018).

5. Khedkar, G.D., Tiknaik, A.D., Shinde, R.N., Kalyankar, A.D., Ron, T.B. and Haymer, D. (2015). High rates of substitution of the native catfish Clarias batrachus by Clarias gariepinus in India. Mitochondrial DNA https://doi.org/10.3109/19401736.2014. 905863

6. Mohanty, B.P., Pati, M.K., Bhattacharjee, S., Hajra, A. and Sharma, A.P. (2013). Small indigenous fishes and their importance in human health. Advances in Fish Research, 5, 257-278.

7. Radhakrishnan, K.V., Lan, Z.J., Zhao, J., Qing, N. and Huang, X.L. (2011). Invasion of the African sharp -tooth catfish Clarias gariepinus (Burchell, 1822) in South China. Biological Invasions, 13, 1723-1727. https://doi.org/10.1007/s10530-011-0004-0

8. Roy, C., Vass, K.K., Patra, B.C., and Sanyal, A.K. (2013). Fish diversity in two south-western districts of West Bengal- Bankura and Purulia. Records of the Zoological Survey of India, $113: 167-179$.

9. Talwar, P.K., and Jhingran, A.G. (1991). Inland fishes of India and adjacent countries. Volume-1. Oxford and IBH Publishing Co. Pvt. Ltd., New Delhi, India. 541. https://trove.nla.gov.au/version/43064856 